\title{
A Comprehensive Classification of Coronaviruses and Inferred Cross-Host Transmissions
}

\author{
Yiran Fu, Marco Pistolozzi, Xiaofeng Yang*, Zhanglin Lin*
}

School of Biology and Biological Engineering, South China University of Technology, 382 East Outer Loop Road, University Park, Guangzhou 510006, China;

* To whom correspondence should be addressed: School of Biology and Biological

Engineering, South China University of Technology, 382 East Outer Loop Road, University Park, Guangzhou 510006, China; Tel: +86-20-3938-0680; Fax: +86-203938-0601; Email: zhanglinlin@scut.edu.cn (Z.L.); biyangxf@scut.edu.cn (X.Y.); 


\begin{abstract}
In this work, we present a unified and robust classification scheme for coronaviruses based on concatenated protein clusters. This subsequently allowed us to infer the apparent "horizontal gene transfer" events via reconciliation with the corresponding gene trees, which we argue can serve as a marker for cross-host transmissions. The cases of SARS-CoV, MERS-CoV, and SARS-CoV-2 are discussed. Our study provides a possible technical route to understand how coronaviruses evolve and are transmitted to humans.
\end{abstract}

\title{
Introduction
}

Coronaviruses (CoVs) are members of the subfamily Orthocoronavirinae in the family Coronaviridae (1), and consist of four genera, i.e., alpha-, beta-, gamma- and deltacoronaviruses, as classified by the International Committee on Taxonomy of Viruses (ICTV) (2-5). All CoVs characterized so far are known to only infect vertebrates, and lead to various zoonotic diseases. Among them, three beta-CoVs are responsible for three major outbreaks of this century (6-10), i.e., the severe acute respiratory syndrome (SARS), the Middle East respiratory syndrome (MERS), and the ongoing global COVID-19 pandemic. Several alpha-CoVs and gamma-CoVs can also greatly affect livestock (11-13). For example, in 2017, the swine acute diarrhea syndrome coronavirus, an alpha-CoV, led to the death of more than 24,000 piglets in Guangdong, China (11).

Characteristic features of CoVs include a high genetic diversity $(7,14)$, frequent 
mutation $(15)$, recombination $(16,17)$, and most importantly, frequent cross-host transmissions $(11,18-20)$ which is facilitated by the spike protein $(21-23)$. These features pose serious challenges for the eradication and mitigation campaigns in case of epidemics, and make even the taxonomic classification of these viruses a challenging task. The traditional classification of CoVs (2-5) relies on phenotypic information such as the host range, and takes into account of the phylogeny of the viruses. Most of these phylogenetic analyses performed on CoVs rely on one of these two approaches (3) : (i) analyses based on the whole genome sequences; (ii) analyses based on individual conserved proteins, most prominently ORF1a/1b, spike, envelope, nucleocapsid, and membrane proteins. These analyses are useful for closely related species/lineages, but become problematic for more distant relationships (24). For example, the node robustness of phylogenetic trees built on the basis of whole genomes of distantly related species is often unreliable as can be judged by their bootstrap values (25). As a result, no comprehensive classification of coronaviruses is currently available.

In this study, we adopted a different approach based on the identification and use of a set of five concatenated CoVs protein clusters to create an Orthocoronavirinae species tree, by using the Markov Cluster Algorithm (MCL, https://www.micans.org/mcl/) (26). This phylogenetic species tree is robust, and is consistent with the current ICTV classification, and thus should be useful for the classification of future new CoV isolates. Furthermore, through reconciliation with the corresponding gene trees (27-29), this species tree allowed us to infer the apparent "horizontal gene transfer" (HGT) events 
for each of the five protein clusters, which we surmised can serve as a marker for crosshost transmissions. The specific cases of SARS-CoV, MERS-CoV, and SARS-CoV-2 are discussed.

\section{Results and Discussion}

We searched through 3,880 genomes of coronaviruses from three databases NCBI, NGDC, and GISAID (https://www.ncbi.nlm.nih.gov/; https://bigd.big.ac.cn/; https://www.gisaid.org), and compiled 396 complete genomes with sequence identity lower than 95\% (Supplementary Table S1). The predicted protein sequences were fed to the subsequent MCL analysis using graph clustering (26). In total, 116 protein clusters were identified, among which five clusters, i.e., ORF1b, ORF1a, spike, membrane, and nucleocapsid proteins were found to be the most prevalent (Table 1, Supplementary Fig. S1). These clusters were then chosen for alignment to construct a bootstrapped phylogenetic tree using Randomized Axelerated Maximum Likelihood (RAxML) (30) (Fig. 1). In this step, we added other 26 genome sequences isolated from different hosts, albeit with identity greater than $95 \%$ to the 396 sequences mentioned above (Supplementary Table S2). The bovine torovirus (NC_007447) from Tobaniviridae $(31,32)$ was selected as the outgroup. The virus genera (according to ICTV classification), the host families and host receptors (if available), and the size of genomes are also included in Fig. 1, in order to provide a comprehensive view of these metadata $(3,4)$. 
As can be seen in Fig. 1, this analysis yields genera and species that are largely congruous with the current ICTV classification (5), but the interpretation of the results is far more straightforward and requires just genome sequences. The resulting phylogenetic tree has a high degree of robustness, compared to those obtained using whole genome sequences, or the ORF1a/1b proteins or domains (see Methods). For example, nodes of branches of the most ancestral lineages of the tree were supported by bootstrap values $\geq 80 \%$. Moreover, this phylogenetic tree can clearly distinguish different species of the viruses for the subfamily Orthocoronavirinae, while the phylogeny based on the ORF1a/b domains alone, which is better suited for classification at the order level (33), cannot do it.

Notably, this circular map format clearly shows the viruses along the same or closely related evolutionary branches can infect different hosts. For instance, in the specific case of SARS-CoV, 29 viruses clustered into a monophyletic clade, which included 22 strains isolated from Chiroptera (bats), four from Viverridae (palm civets), and three from Hominidae (humans). Similarly, several MERS-CoVs that form a monophyletic clade in the inferred tree were isolated from Chiroptera, Camelidae (dromedary camels), and Hominidae. In the section containing the coronaviruses Human_CoV_OC43 which are responsible for a common human flu, several hosts appear in the map $(34,35)$, reflecting the considerable promiscuity of their hosts including Bovidae (cattle), Leporidae (rabbits), Camelidae, Suidae (swine), and Equidae (horses). 
We next determined the apparent HGT events among the viruses, which we assumed would indicate their cross-host transmissions. To this end, the reconciliations between the concatenated phylogeny and the related gene trees for the five protein clusters was performed, using RANGER-DTL version 2.0 (36). A visualized summary is given in Fig. 2 (30). We identified three types of transfer: (i) clade to clade, (ii) clade to strain, (iii) strain to strain. Within the same host, HGT occurred most frequently for Phasianidae, followed by Felidae and Hominidae (Fig. 2B). Transfer events across different hosts for clusters 3, 4, 5 (spike, membrane, and nucleocapsid proteins, respectively) were more frequent than for the other two clusters. Among different hosts, bats were the most frequent donor or receiver, followed by Suidae, Camelidae, Hominidae, and Phasianidae (Fig. 2C). This immediately suggests an urgent need for erecting ecological barriers between livestock and wild animals, and between humans and wild animals, as well as for worldwide monitoring of coronaviruses.

We then examined in more details the inferred HGT events for SARS-CoV, MERS$\mathrm{CoV}$ and SARS-CoV-2. For this analysis, an expanded dataset of 269 complete genomes of the beta-CoVs with sequence identity lower than $99.8 \%$ was used, except that only four SARS-CoV-2 genomes of human origin were included (37), to reduce the redundancy (Supplementary Table S3). The Human_CoV_229E (NC_002645) from alpha-CoVs (3) was selected as the outgroup. For SARS-CoV (Fig. 3A), there were apparent transfer links among Chiroptera, Viverridae and Hominidae. This result is consistent with the current knowledge concerning the evolution of SARS-CoVs, with 
the palm civet as the likely intermediate host $(16,38,39)$. As there is no evidence for direct transmission of this virus between Chiroptera and Hominidae, the gene transfer between Hominidae and Chiroptera as depicted in Fig. 3A might be an indirect result of separated transfer events between Chiroptera and Viverridae, and between Viverridae and Hominidae. The pattern found for MERS-CoV (Fig. 3B) is again consistent with accumulated evidence indicating the dromedary camel as a probable vector for human infections $(8,40,41)$. For SARS-CoV-2 (Fig. 3C), transfer links were found between Chiroptera and Hominidae, and between Chiroptera and Manidae (pangolins). Interestingly, no gene transfer between Hominidae and Manidae can be inferred from our analysis. Pangolins were suggested to be involved in the species jump of SARS-CoV-2 to humans $(37,42)$. However, more recent studies have disputed this notion (37, 43, 44). Finally, while the links among Hominidae, Mustelidae (minks), and Felidae (tigers) were deduced, it remains to be seen how SARS-CoV-2 viruses were transmitted among them. Taken together, we argue that these inferred apparent HGT events can serve as a possible marker for cross-host transmissions of coronaviruses.

In summary, based on the analysis of concatenated protein clusters of the viral genome sequences, we present a framework to produce robust phylogeny and classification for CoVs, and to infer the apparent HGT events and cross host transmissions for the Orthocoronavirinae subfamily. This should help to understand how CoVs are transmitted to humans, and aid in devising strategies to combat the severe global health threat posed by these viruses. 


\section{Methods}

\section{Genome sequence collection and marker selection}

Genome datasets were compiled with CD-HIT version 4.7 (45). Open reading frames (ORFs) in the datasets were predicted by using GeneMarkS version 4.32 (46), and then annotated using against the NR database (https://ftp.ncbi.nlm.nih.gov/blast/db/FASTA/) with e-value $\leq 10^{-5}$.

Marker selections were performed with the Markov Cluster algorithm of OrthoMCL, with the parameter of '-I 1.5 ' $(26)$. The 20 protein clusters with abundancy in a genus higher than 30\% are listed in Supplementary Fig. S1.

\section{Phylogenetic inference and apparent HGT inference}

For the phylogenetic analyses, the multiple sequence alignments (MSAs) of the datasets (the 422 genome sequences used for the analysis of the subfamily Orthocoronavirinae, and the 269 genome sequences used for the analysis of beta-CoVs ) were analyzed by MAFFT v7.407 (47) based on the five concatenated protein clusters or each individual cluster. Subsequently, maximum likelihood phylogenies were estimated using RAxML version 7.2.8 (48), utilizing the PROTGAMMALG model with 100 bootstrap replicates. The apparent HGT events were inferred based on reconciliation of the species tree and the corresponding gene tree (27), by using RANGER-DTL version 2.0 with default parameters (https://compbio.engr.uconn.edu/software/RANGER-DTL/) $(36,49)$. The apparent HGT events were then presented by using Gephi 0.9.2.

For comparison of robustness, two other phylogenetic trees were also built, based on 
the full genome, and the five concatenated domains of ORF1a/b that are used in the ICTV classification approach by the Coronaviridae Study Group (e.g., 3CLpro, NiRAN, RdRp, ZBD, and HEL1) $(3,33)$. The phylogenetic tree based on the full genome was constructed using RAxML with the GTRGAMMA model and 1,000 bootstrap replicates. The phylogenetic tree based on the five concatenated domains of ORF1a/1b was built using the same method as that based on the five concatenated protein clusters described above.

\section{References}

1. J. Cui, F. Li, Z. L. Shi, Origin and evolution of pathogenic coronaviruses. Nat Rev Microbiol 17, 181-192 (2019).

2. L. Laenen et al., Hantaviridae: Current classification and future perspectives. Viruses 11, 788 (2019).

3. Coronaviridae Study Group of the International Committee on Taxonomy of Viruses, The species Severe acute respiratory syndrome-related coronavirus: classifying 2019-nCoV and naming it SARS-CoV-2. Nat Microbiol 5, 536-544 (2020).

4. P. Simmonds, Methods for virus classification and the challenge of incorporating metagenomic sequence data. J Gen Virol 96, 1193-1206 (2015).

5. International Committee on Taxonomy of Viruses Executive Committee, The new scope of virus taxonomy: partitioning the virosphere into 15 hierarchical ranks. Nat Microbiol 5, 668-674 (2020). 
6. P. Zhou et al., A pneumonia outbreak associated with a new coronavirus of probable bat origin. Nature 579, 270-273 (2020).

7. M. Letko, S. N. Seifert, K. J. Olival, R. K. Plowright, V. J. Munster, Bat-borne virus diversity, spillover and emergence. Nat Rev Microbiol 18, 461-471 (2020).

8. D. K. W. Chu et al., MERS coronaviruses from camels in Africa exhibit regiondependent genetic diversity. Proc Natl Acad Sci U S A 115, 3144-3149 (2018).

9. O. W. Ng, Y. J. Tan, Understanding bat SARS-like coronaviruses for the preparation of future coronavirus outbreaks - Implications for coronavirus vaccine development. Hum Vaccin Immunother 13, 186-189 (2017).

10. J. ter Meulen et al., Human monoclonal antibody combination against SARS coronavirus: synergy and coverage of escape mutants. PLoS Med 3, e237 (2006).

11. P. Zhou et al., Fatal swine acute diarrhoea syndrome caused by an HKU2-related coronavirus of bat origin. Nature 556, 255-258 (2018).

12. L. M. Parsons et al., Glycosylation of the viral attachment protein of avian coronavirus is essential for host cell and receptor binding. J Biol Chem 294, 7797-7809 (2019).

13. H. Rezaee et al., Molecular detection of avian infectious bronchitis viruses in live bird markets, Gilan Province. Arch Razi Inst 75, 155-162 (2020).

14. X. C. Tang et al., Prevalence and genetic diversity of coronaviruses in bats from China. J Virol 80, 7481-7490 (2006).

15. V. B. Vega et al., Mutational dynamics of the SARS coronavirus in cell culture and human populations isolated in 2003. BMC Infect Dis 4, 32 (2004). 
16. B. Hu et al., Discovery of a rich gene pool of bat SARS-related coronaviruses provides new insights into the origin of SARS coronavirus. PLoS Pathog 13, e1006698 (2017).

17. A. D. Regan et al., Characterization of a recombinant canine coronavirus with a distinct receptor-binding (S1) domain. Virology 430, 90-99 (2012).

18. Q. Li et al., Early transmission dynamics in Wuhan, China, of novel coronavirus-infected pneumonia. N Engl J Med 382, 1199-1207 (2020).

19. G. Lu, Q. Wang, G. F. Gao, Bat-to-human: spike features determining 'host jump' of coronaviruses SARS-CoV, MERS-CoV, and beyond. Trends Microbiol 23, 468-478 (2015).

20. B. L. Haagmans et al., Middle East respiratory syndrome coronavirus in dromedary camels: an outbreak investigation. Lancet Infect Dis 14, 140-145 (2014).

21. Y. Wang et al., A recombinant infectious bronchitis virus from a chicken with a spike gene closely related to that of a turkey coronavirus. Arch Virol 165, 703707 (2020).

22. X. Zhang et al., Identification of a natural recombinant transmissible gastroenteritis virus between Purdue and Miller clusters in China. Emerg Microbes Infect 6, e74 (2017).

23. X. Wang et al., Co-circulation of canine coronavirus I and IIa/b with high prevalence and genetic diversity in Heilongjiang Province, Northeast China. PLoS One 11, e0146975 (2016). 
24. R. T. Y. So et al., Diversity of dromedary camel coronavirus HKU23 in African camels revealed multiple recombination events among closely related betacoronaviruses of the subgenus embecovirus. J Virol 93, e01236-01219 (2019).

25. S. J. Low, M. Džunková, P.-A. Chaumeil, D. H. Parks, P. Hugenholtz, Evaluation of a concatenated protein phylogeny for classification of tailed double-stranded DNA viruses belonging to the order Caudovirales. Nature Microbiology 4, 1306-1315 (2019).

26. L. Li, C. J. Stoeckert, Jr., D. S. Roos, OrthoMCL: identification of ortholog groups for eukaryotic genomes. Genome Res 13, 2178-2189 (2003).

27. M. Ravenhall, N. Skunca, F. Lassalle, C. Dessimoz, Inferring horizontal gene transfer. PLoS Comput Biol 11, e1004095 (2015).

28. C. Than, D. Ruths, H. Innan, L. Nakhleh, Confounding factors in HGT detection: statistical error, coalescent effects, and multiple solutions. J Comput Biol 14, $517-535$ (2007).

29. M. Hellmuth, N. Wieseke, in Evolutionary Biology. (2016), chap. Chapter 21, pp. 373-392.

30. M. Bastian, Gephi : An open source software for exploring and manipulating networks. International AAAI Conference on Weblogs and Social Media, (2009).

31. L. M. Duckmanton, R. Tellier, P. Liu, M. Petric, Bovine torovirus: sequencing of the structural genes and expression of the nucleocapsid protein of Breda virus. 
Virus Res 58, 83-96 (1998).

32. J. M. Gonzalez, P. Gomez-Puertas, D. Cavanagh, A. E. Gorbalenya, L. Enjuanes, A comparative sequence analysis to revise the current taxonomy of the family Coronaviridae. Arch Virol 148, 2207-2235 (2003).

33. A. Saberi, A. A. Gulyaeva, J. L. Brubacher, P. A. Newmark, A. E. Gorbalenya, A planarian nidovirus expands the limits of RNA genome size. PLoS Pathog 14, e1007314 (2018).

34. S. Su et al., Epidemiology, genetic recombination, and pathogenesis of coronaviruses. Trends Microbiol 24, 490-502 (2016).

35. D. Forni, R. Cagliani, M. Clerici, M. Sironi, Molecular evolution of human coronavirus genomes. Trends Microbiol 25, 35-48 (2017).

36. M. S. Bansal, E. J. Alm, M. Kellis, Efficient algorithms for the reconciliation problem with gene duplication, horizontal transfer and loss. Bioinformatics 28, i283-i291 (2012).

37. T. T. Lam et al., Identifying SARS-CoV-2-related coronaviruses in Malayan pangolins. Nature 583, 282-285 (2020).

38. W. H. Li et al., Angiotensin-converting enzyme 2 is a functional receptor for the SARS coronavirus. Nature 426, 450-454 (2003).

39. V. D. Menachery et al., A SARS-like cluster of circulating bat coronaviruses shows potential for human emergence. Nat Med 21, 1508-1513 (2015).

40. J. S. M. Sabir, Co-circulation of three camel coronavirus species and recombination of MERS-CoVs in Saudi Arabia. Science 351, 81-84 (2016). 
41. J. Rodon et al., Blocking transmission of Middle East respiratory syndrome coronavirus (MERS-CoV) in llamas by vaccination with a recombinant spike protein. Emerg Microbes Infect 8, 1593-1603 (2019).

42. T. Zhang, Q. Wu, Z. Zhang, Probable pangolin origin of SARS-CoV-2 associated with the COVID-19 outbreak. Curr Biol 30, 1346-1351 (2020).

43. G. Z. Han, Pangolins harbor SARS-CoV-2-related coronaviruses. Trends Microbiol 28, 515-517 (2020).

44. P. Liu et al., Are pangolins the intermediate host of the 2019 novel coronavirus (SARS-CoV-2)? PLoS Pathog 16, e1008421 (2020).

45. W. Li, A. Godzik, Cd-hit: a fast program for clustering and comparing large sets of protein or nucleotide sequences. Bioinformatics 22, 1658-1659 (2006).

46. J. Besemer, A. Lomsadze, M. Borodovsky, GeneMarkS: a self-training method for prediction of gene starts in microbial genomes. Implications for finding sequence motifs in regulatory regions. Nucleic Acids Res 29, 2607-2618 (2001).

47. J. Rozewicki, S. Li, K. M. Amada, D. M. Standley, K. Katoh, MAFFT-DASH: integrated protein sequence and structural alignment. Nucleic Acids Res 47, W5W10 (2019).

48. A. Stamatakis, RAxML version 8: a tool for phylogenetic analysis and postanalysis of large phylogenies. Bioinformatics 30, 1312-1313 (2014).

49. L. Nakhleh, Computational approaches to species phylogeny inference and gene tree reconciliation. Trends Ecol Evol 28, 719-728 (2013).

50. I. Letunic, P. Bork, Interactive tree of life (iTOL) v3: an online tool for the 
bioRxiv preprint doi: https://doi.org/10.1101/2020.08.11.232520; this version posted August 11, 2020. The copyright holder for this preprint (which was not certified by peer review) is the author/funder, who has granted bioRxiv a license to display the preprint in perpetuity. It is made available under aCC-BY-NC 4.0 International license.

display and annotation of phylogenetic and other trees. Nucleic Acids Res 44,

W242-W245 (2016). 


\section{Figure legends}

Figure 1. Rooted phylogeny of 422 Orthocoronavirinae viruses with the bovine torovirus from Tobaniviridae as the outgroup, inferred from five concatenated protein clusters using RAxML v7.2.8. Bootstrap support values $\geq 80 \%$ are shown as dots on interior nodes. Ring colors indicate the virus genus classification (indicated as squares in the legend) and their host phylogeny (indicated as triangles in the legend). Virus genome sizes are shown in the peripheral circle. Coronavirus information is provided in Supplementary Tables S1/S2. The visualization of the phylogenetic tree was performed by using iToL (https://itol.embl.de/) (50).

Figure 2. Statistics of the inferred horizontal gene transfer (HGT) events and host jumps of coronaviruses. A. Counts of transfer events that are within the same host family and among different host families. The colors within a bar stand for three different type of transfer events (i.e., from clade to clade, from clade to strain, from strain to strain. B. Counts of transfer events within the same host family. Each circle indicates a host family as shown in the label and its size is proportional to the number of transfer events within the same host family. C. Inferred host jump network of coronaviruses based on the transfer events. Each line indicates the predicted host jump between two host families and its thickness is proportional to the number of host jump events. Each circle indicates host family as shown in the label and its size is proportional to the number of host jump between different host families. (Figs. 2B and 2C is generated using Gephi 0.9.2, https://gephi.org) (30). 
Figure 3. Cross-host transmissions inferred from horizontal gene transfer (HGT) events for SARS-CoV (A), MERS-CoV (B), and SARS-CoV-2 (C). The circles correspond to the hosts, and the lines correspond to the apparent HGT events among the hosts. 


\section{Tables}

Table 1. The five protein clusters as the single-copy markers of the subfamily

Orthocoronavirinae.

\begin{tabular}{cccc}
\hline Cluster Id & $\begin{array}{c}\text { Number of } \\
\text { Single Copy Gene }\end{array}$ & $\begin{array}{c}\text { Average } \\
\text { Length (aa) }\end{array}$ & $\begin{array}{c}\text { Cluster Protein } \\
\text { Annotation }\end{array}$ \\
\hline Cluster1 & 397 & 2612 & ORF1b Protein \\
Cluster2 & 396 & 4103 & ORF1a Protein \\
Cluster3 & 396 & 1285 & Spike Protein \\
Cluster4 & 396 & 229 & Membrane Protein \\
Cluster5 & 396 & 405 & Nucleocapsid Protein \\
\hline
\end{tabular}




\section{Host}

$\checkmark$ Phasianidae

$\checkmark$ Clairoptera:

Hominida

$\triangle$ Felidae

$\triangle$ Muridae

$\triangleright$ Camelidae

$\checkmark$ Canidae

Mustelidae

$\triangle$ Bovidae

$\triangleright$ Erinaceidae

$\triangleright$ Anatidae

$\triangle$ Viverridae

Cervidae
Manidae

$\triangle$ Leporidae

$\triangleright$ Delphinidae

$\triangleright$ Cetacea

$\triangle$ Equidae

$>$ Giraffidae

$\checkmark$ Cercopithecidae

$\checkmark$ Passeridae

$\checkmark$ Falconidae

$\triangle$ Zosteropidae

$>$ Turdidae

$\checkmark$ Pycnonotidae

$\triangle$ Ploceidae

$\checkmark$ Muscicapidae

$\checkmark$ Ardeidae

$\checkmark$ Rallidae

\section{Genome size}

Minimum (25380bp); maximum (31775bp)

6 Events

7 events related to humans disease

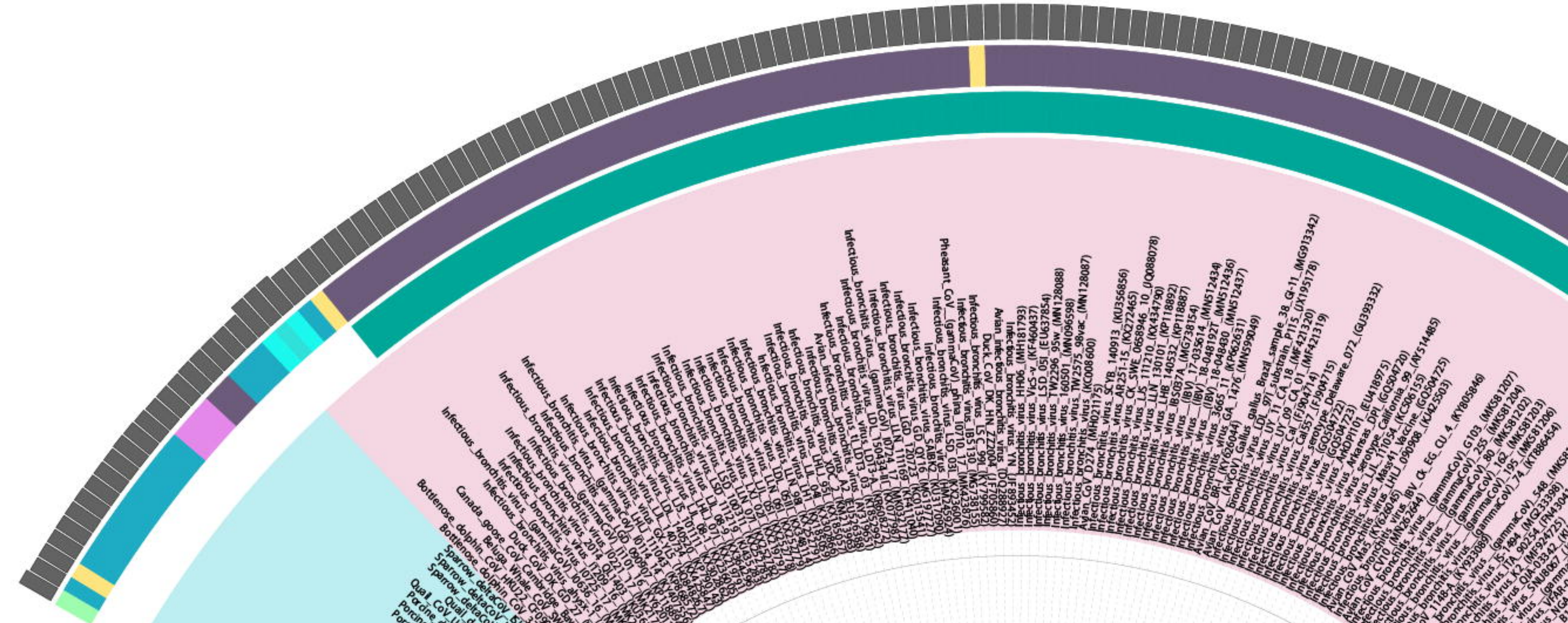
$s^{2}$

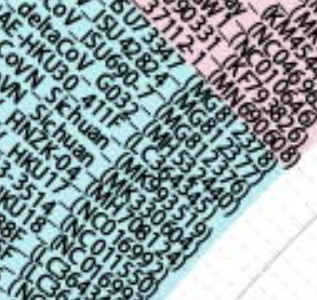

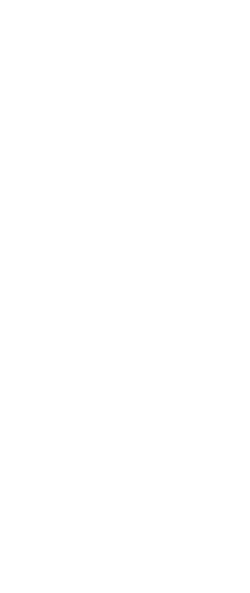
,

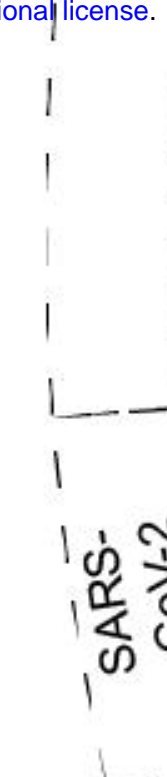

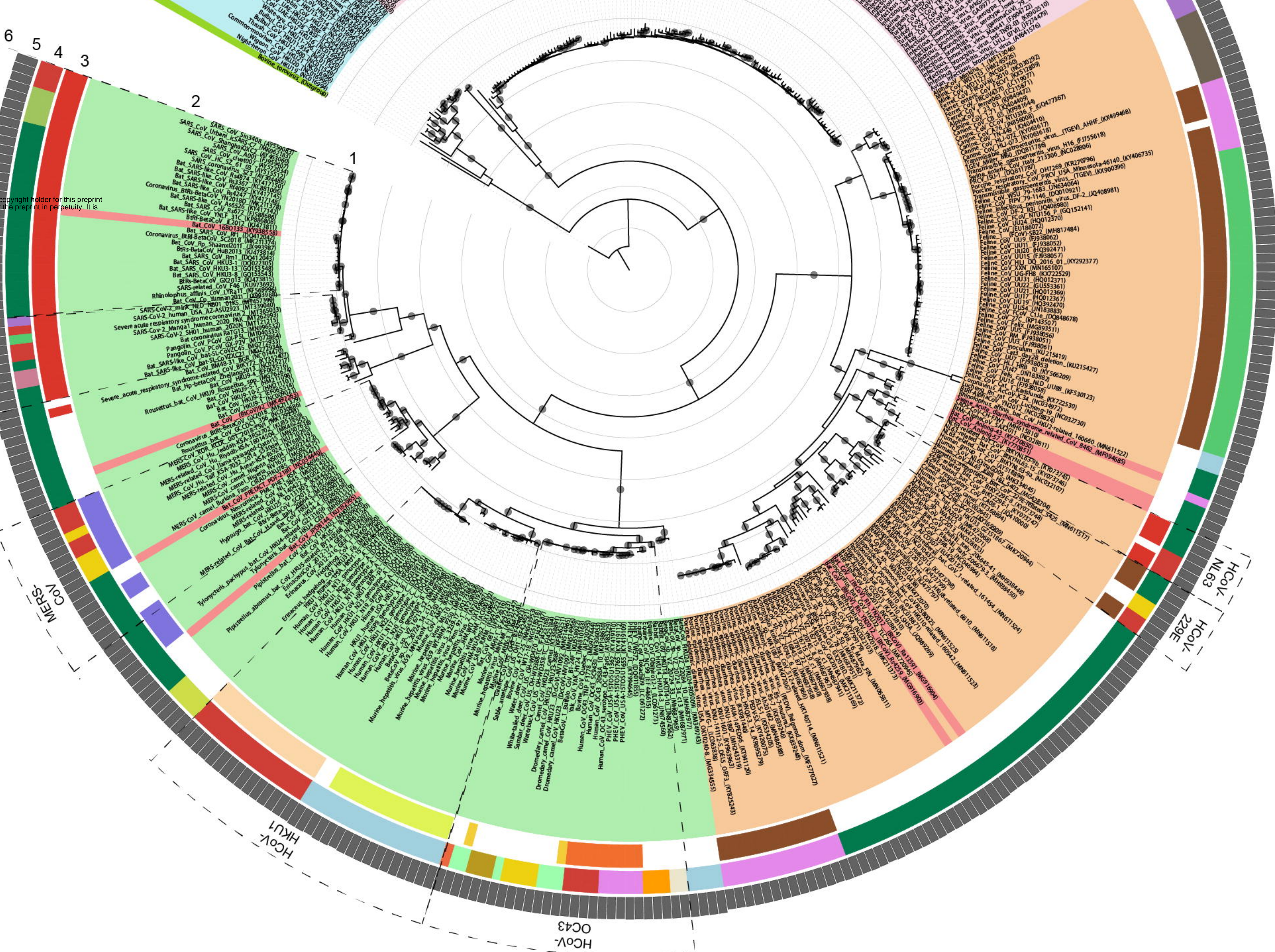


A

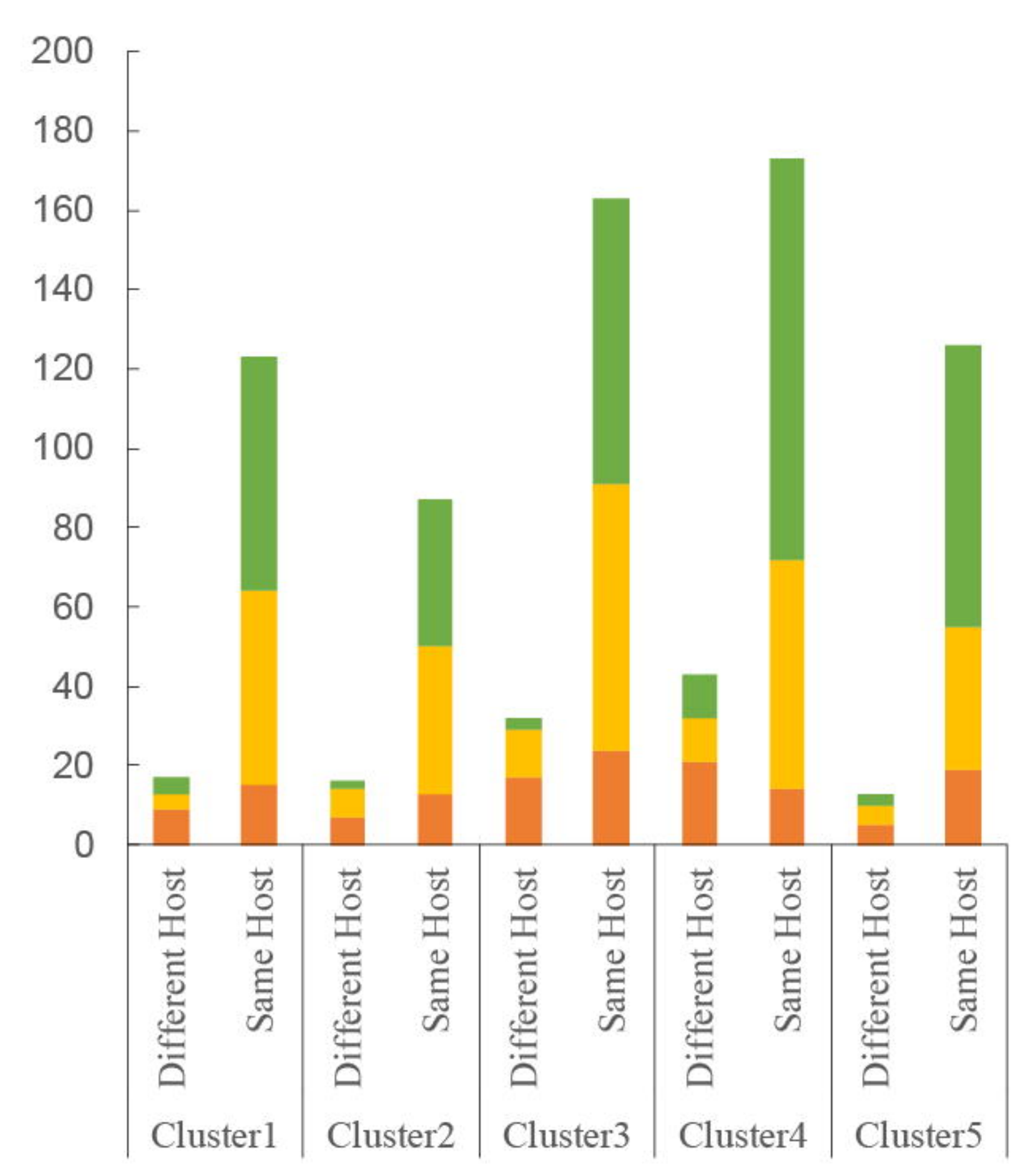

Class1 Clade to Clade

Class2 Clade to Strain

- Class3 Strain to Strain

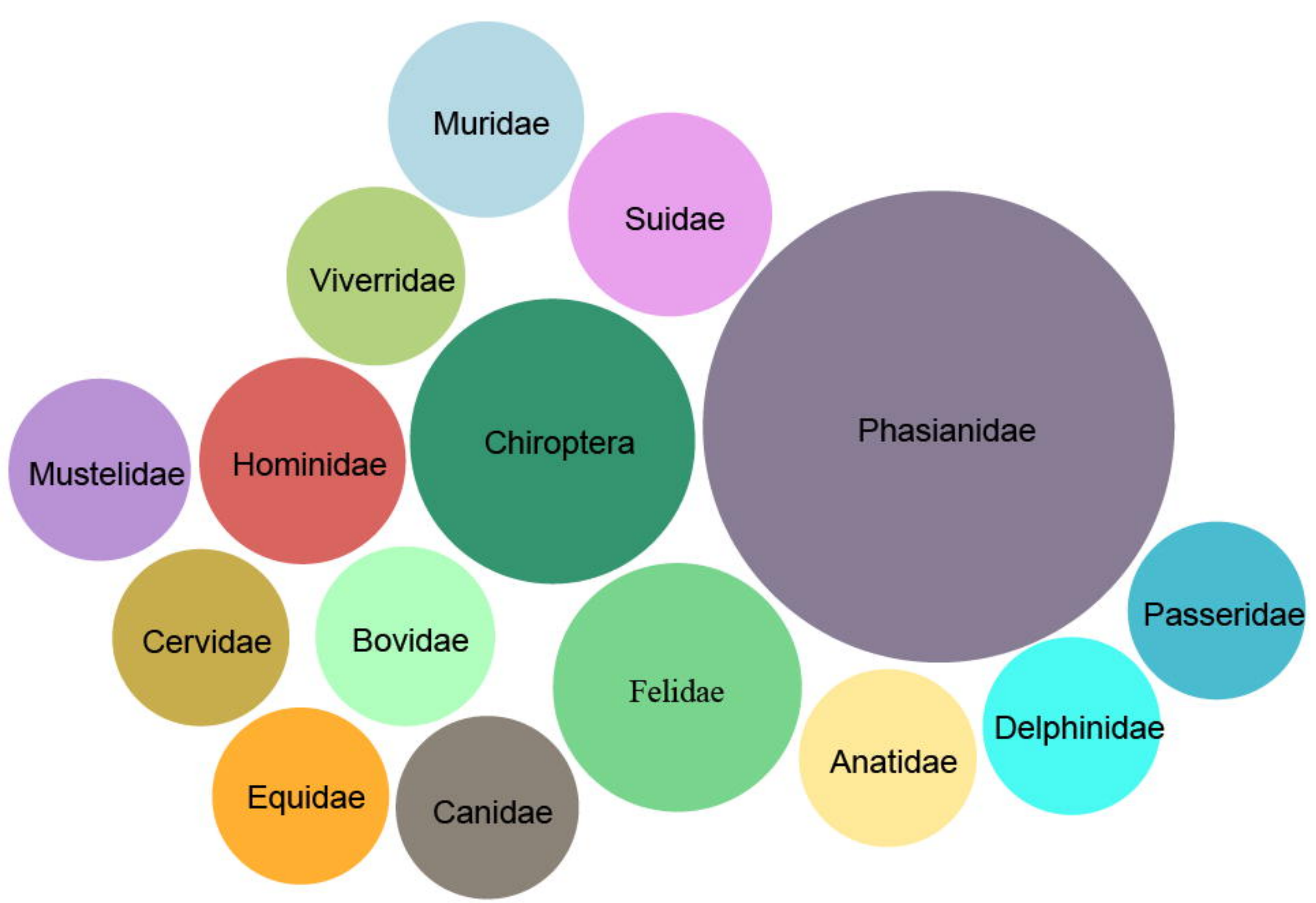

C

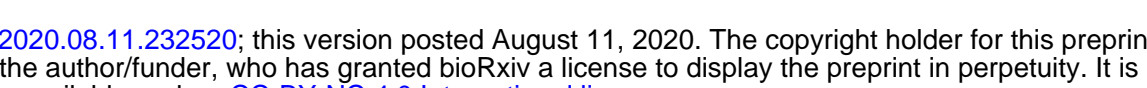

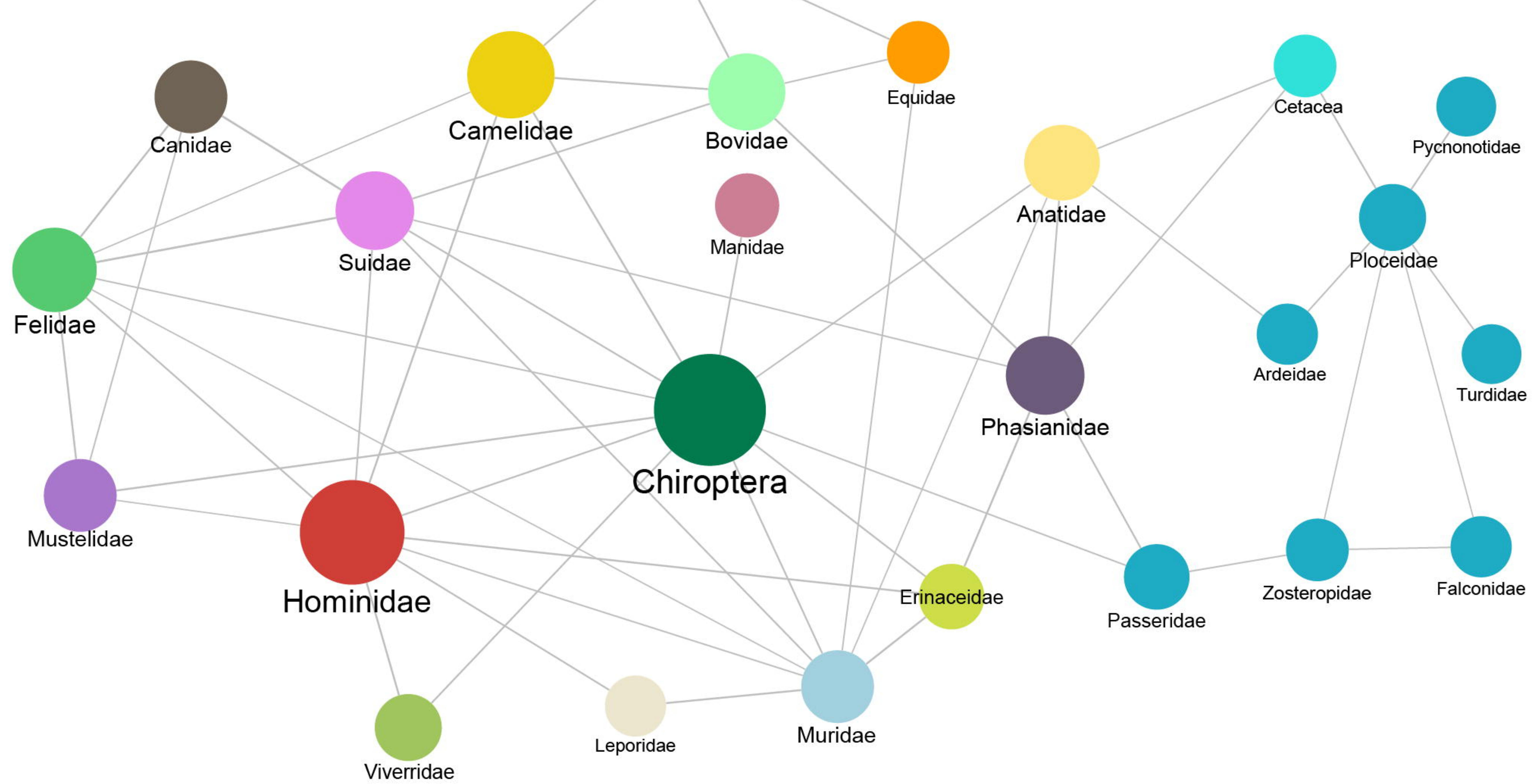




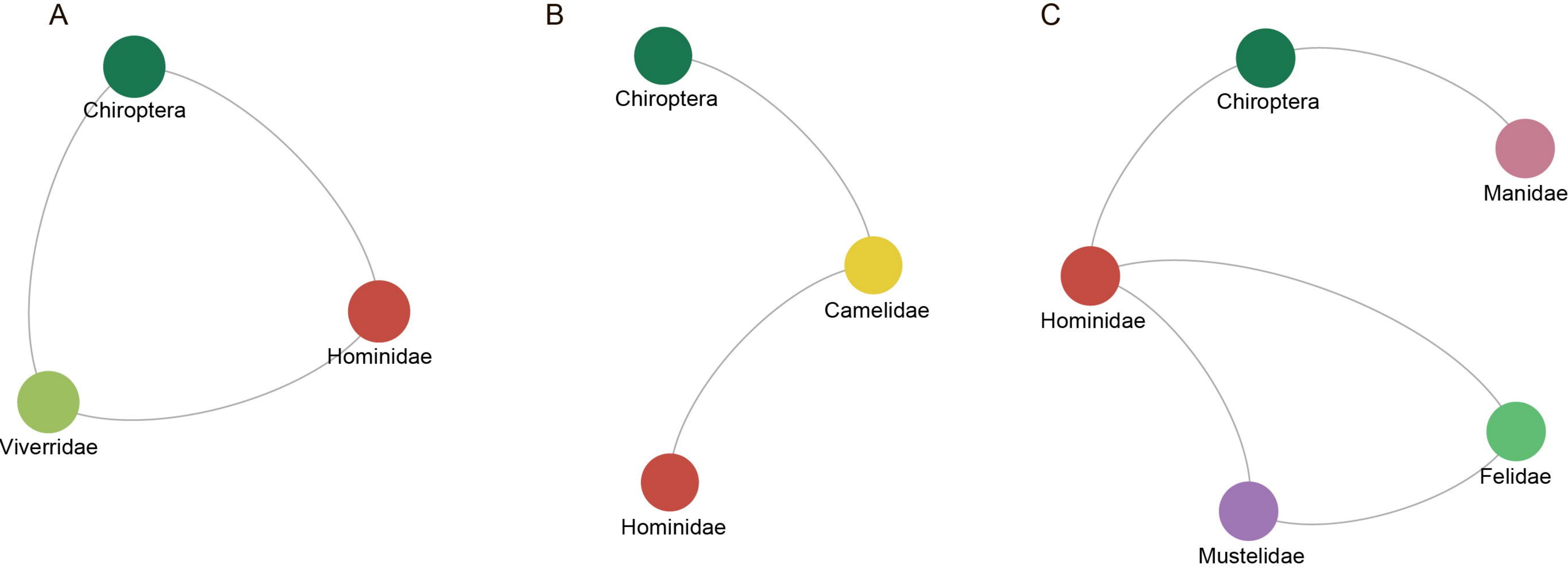

